\title{
THE DIFFERENTIABILITY OF THE DRAG WITH RESPECT TO THE VARIATIONS OF A LIPSCHITZ DOMAIN IN A NAVIER-STOKES FLOW*
}

\author{
JUAN ANTONIO BELLO ${ }^{\dagger}$, ENRIQUE FERNÁNDEZ-CARA ${ }^{\dagger}$, JÉRÔME LEMOINE JAND $^{\ddagger}$ \\ JACQUES SIMON ${ }^{\ddagger}$
}

\begin{abstract}
This paper is concerned with the computation of the drag $T$ associated with a body traveling at uniform velocity in a fluid governed by the stationary Navier-Stokes equations. It is assumed that the fluid fills a domain of the form $\Omega+u$, where $\Omega \subset \mathbb{R}^{3}$ is a reference domain and $u$ is a displacement field. We assume only that $\Omega$ is a Lipschitz domain and that $u$ is Lipschitz-continuous. We prove that, at least when the velocity of the body is sufficiently small, $u \mapsto T(\Omega+u)$ is a $C^{\infty}$ mapping (in a ball centered at 0 ). We also compute the derivative at 0 .
\end{abstract}

Key words. domain optimization, hydrodynamic drag, Navier-Stokes equations, Lipschitz domains, optimal control

AMS subject classification. 49J20

PII. S0363012994278213

1. Introduction. Formulation of the problem. In this paper, we study the behavior of the drag $T$ associated with a body traveling at uniform velocity $\gamma$ in a viscous incompressible fluid. It is assumed that the flow of this fluid is governed by the stationary Navier-Stokes equations. We are interested in viewing $T$ as a function of the shape of the body.

More precisely, let $B$ be a reference shape for the body and $\Omega$ be the corresponding fluid domain. The body variations are described by a field $u$, and we search for a formula of the kind

$$
T(\Omega+u)=T(\Omega)+T^{\prime}(\Omega ; u)+o(u),
$$

where the modified fluid domain is

$$
\Omega+u=\left\{x \in \mathbb{R}^{d} ; x=(I+u)(\xi), \xi \in \Omega\right\} .
$$

We are thus led to an analysis of the differentiability of the function $u \mapsto T(\Omega+u)$.

The main results. We prove that when $\Omega$ is a Lipschitz domain, $u$ is Lipschitzcontinuous, and the velocity $\gamma$ is sufficiently small, the function $u \mapsto T(\Omega+u)$ is differentiable. More precisely (see Theorem 4 ), we show that it is a $C^{\infty}$ mapping in a small ball $\mathcal{W}$ whose elements are Lipschitz vector fields. We also compute explicitly $T^{\prime}(\Omega ; u)$, i.e., the derivative at 0 in the direction $u$.

In the similar but more simple case of an elliptic equation, differentiability results have been established by F. Murat and J. Simon in [9], [10] without any regularity hypothesis on $\Omega$. The proof relies on the change of variables $x=(I+u)(\xi)$, by means of which one is led to a fixed domain. This method has been used for many equations by several authors.

${ }^{*}$ Received by the editors December 5, 1994; accepted for publication (in revised form) February 20, 1996.

http://www.siam.org/journals/sicon/35-2/27821.html

${ }^{\dagger}$ Departamento de Ecuaciones Diferenciales y Análisis Numérico, Universidad de Sevilla, Aptdo. 1160, 41080 Sevilla, Spain. The research of these authors was supported in part by Proyecto Dirección General de Investigación Cientifica y Tecnologica.

${ }^{\ddagger}$ Laboratoire de Mathématiques Appliquées, Université Blaise Pascal (Clermont-Ferrand 2), 63177 Aubière Cedex, France (simon@ucfma.univ-bpclermont.fr). 
Some difficulties related to incompressibility. The general method in [9], [10] cannot be directly applied to the Stokes and Navier-Stokes cases. This is due to the incompressibility condition

$$
\nabla \cdot y(u)=0 \quad \text { in } \Omega+u,
$$

which has to be satisfied by the velocity field $y(u)$. This difficulty was surmounted when $\Omega$ is a $W^{2, \infty}$ domain by J. Simon [17] for Stokes flows and by J. A. Bello, E. Fernández-Cara, and J. Simon [1], [2] for Navier-Stokes flows. In [17], the author uses a variant of the implicit function theorem; in [1], [2], one introduces a family of isomorphisms which allow us to rewrite the equation $\nabla \cdot y(u)=0$ appropriately. In this paper, the incompressibility equation is rewritten explicitly.

We will assume that $\Omega$ is a Lipschitz domain and that $u$ is Lipschitz-continuous. This includes many interesting situations in which $\partial \Omega$ and/or $\partial(\Omega+u)$ possess "corner" points.

Recall that formal computations of the derivative were previously carried out by O. Pironneau [12] (see also [13]) using "normal" variations.

Some difficulties related to weak regularity. The "natural" expression of the derivative $T^{\prime}(\Omega ; u)$ (that is, the right-hand side of (15)) is not defined a priori since $y$ is only $H^{1}(\Omega)^{d}$. Nevertheless, we will give a meaning for this expression using the technical result (17).

2. The definition of the drag. Let $D$ and $B$ be bounded open connected sets in $\mathbb{R}^{d}, d=2$ or 3 , with $B \subset \subset D$. Let us set $\Omega=D \backslash \bar{B}$. In the following discussion, it will be assumed that

$$
\Omega \text { is a Lipschitz domain; }
$$

that is to say, its boundary $\partial \Omega$ is locally the graph of a Lipschitz-continuous function and $\Omega$ is the corresponding epigraph. (This is explained more in detail in the appendix.) $[4]$

Let $\gamma \in \mathbb{R}^{d}$ be a given vector. We consider the stationary Navier-Stokes problem

$$
\left\{\begin{array}{l}
y-g \in H_{0}^{1}(\Omega)^{d} \\
p \in L^{2}(\Omega), \quad \int_{\Omega} p=0 \\
-\nu \Delta y+(y \cdot \nabla) y+\nabla p=0 \\
\nabla \cdot y=0
\end{array}\right.
$$

Here, $g \in H^{1}\left(\mathbb{R}^{d}\right)^{d}$ and satisfies

(3) $\nabla \cdot g=0, g=\gamma$ in a neighborhood of $\partial D, g=0$ in a neighborhood of $B$.

When $B$ is small with respect to $D$, any solution $(y, p)$ to $(2)$ provides good approximations to the velocity field and the pressure distribution of a viscous incompressible fluid in $\Omega$ having constant velocity far from $B$. It can be imagined that we have chosen spatial coordinates fixed with respect to $B, D$ is an approximation to $\mathbb{R}^{d}$, the fluid is at rest at infinity, and $B$ is the shape of a body traveling at constant velocity $-\gamma$.

The requirement $\int_{\Omega} p=0$ provides uniqueness for the pressure $p$ that, otherwise, would be defined up to an additive constant. 
If $\gamma$ is sufficiently small, problem (2) possesses exactly one solution, which is "small" and does not depend on the choice of $g$. More precisely, Theorem 2.1 in [9] gives the following lemma.

LEMMA 1. There exists a constant $\alpha>0$ such that, if $|\gamma|<\alpha \nu$, then (2) possesses exactly one solution, $(y, p) \in H^{1}(\Omega)^{d} \times L^{2}(\Omega)$. This solution does not depend on the choice of the function $g$ satisfying (3). Furthermore, for each $\epsilon>0$, the constant $\alpha$ can be chosen in such a way that

$$
\|y\|_{H^{1}(\Omega)^{d}} \leq \epsilon \nu .
$$

If $\mathcal{O} \subset \subset D$ is given, one can also choose $\alpha=\alpha(\epsilon, \mathcal{O}, D)$ not depending on $B$, provided $B \subset \mathcal{O}$. Finally, if $\Omega$ is a $W^{2, \infty}$ domain, then $(y, p) \in H^{2}(\Omega)^{d} \times H^{1}(\Omega)$.

Thus, at least when $\gamma$ is small, one can associate with $\Omega$ a drag

$$
T(\Omega)=\frac{\nu}{2} \int_{\Omega} \sigma(y)^{2},
$$

where $\sigma(y)^{2}=\sigma(y) \cdot \sigma(y) \equiv \sum_{i j}\left(\sigma_{i j}(y)\right)^{2}$.

Remark. If $\Omega$ is regular enough, $T(\Omega)$ coincides with the usual hydrodynamical drag, which is given as follows (cf. [14]):

$$
\mathcal{T}(\Omega)=-\gamma \cdot \int_{\partial B}(-p I d+\nu \sigma(y)) \cdot n d s
$$

Indeed, using the boundary condition, we obtain

$$
\mathcal{T}(\Omega)=-\int_{\partial \Omega}(p(y-\gamma)-\nu \sigma(y) \cdot(y-\gamma)) \cdot n d s .
$$

From Gauss formula and incompressibility, this gives

$$
\begin{aligned}
\mathcal{T}(\Omega) & =-\int_{\Omega} \nabla \cdot(p(y-\gamma)-\nu \sigma(y) \cdot(y-\gamma)) \\
& =\int_{\Omega}((\nu \Delta y-\nabla p) \cdot(y-\gamma)+\nu \sigma(y) \cdot \nabla y) .
\end{aligned}
$$

Note that, again using incompressibility,

$$
(\nu \Delta y-\nabla p) \cdot(y-\gamma)=((y \cdot \nabla) y) \cdot(y-\gamma)=\nabla \cdot\left(|y-\gamma|^{2} y\right) .
$$

Therefore,

$$
\int_{\Omega}(\nu \Delta y-\nabla p) \cdot(y-\gamma)=\int_{\partial \Omega}|y-\gamma|^{2} y \cdot n d s=0,
$$

and, finally, since $\sigma(y) \cdot \nabla y=\frac{1}{2} \sigma(y)^{2}$, we have $\mathcal{T}(\Omega)=T(\Omega)$.

3. The domain variations. We will choose fields $u \in W^{1, \infty}\left(\mathbb{R}^{d}, \mathbb{R}^{d}\right)$ such that $u=0$ on $\partial D$. This condition expresses the fact that the outer boundary limiting the fluid is fixed.

We will also assume $\|u\|_{\text {Lip }}<c(\Omega)$, with $c(\Omega)$ being small enough to ensure that $\Omega+u$ is Lipschitzian and also that $B+u$ is included in a fixed open set $\mathcal{O}$ satisfying

$$
B \subset \subset \mathcal{O} \subset \subset D
$$


Here, we have denoted by $\|u\|_{\text {Lip }}$ the best Lipschitz constant for $u$. More precisely, we have the following obvious result (see [8] for a proof).

Lemma 2. Assume that $\mathcal{O}$ is as before. There exists $c(\Omega), 0<c(\Omega)<1$, such that

$$
B+u \subset \mathcal{O}
$$

for all $u \in W^{1, \infty}\left(\mathbb{R}^{d}, \mathbb{R}^{d}\right)$ satisfying $u=0$ on $\partial D$ and $\|u\|_{\text {Lip }} \leq c(\Omega)$.

We will also use the following result, whose proof is given in the appendix.

LEMMA 3. There exists $c(\Omega), 0<c(\Omega)<1$, such that

$$
\Omega+u \text { is a bounded Lipschitz domain in } \mathbb{R}^{d}
$$

for all $u \in W^{1, \infty}\left(\mathbb{R}^{d}, \mathbb{R}^{d}\right)$ satisfying $\|u\|_{W^{1, \infty}\left(\mathbb{R}^{d}, \mathbb{R}^{d}\right)} \leq c(\Omega)$.

Remark. This lemma holds for each bounded Lipschitz domain $\Omega \subset \mathbb{R}^{d}$.

For the subsequent discussion, we introduce

$$
\mathcal{W}=\left\{u \in W^{1, \infty}\left(\mathbb{R}^{d}, \mathbb{R}^{d}\right) ;\|u\|_{W^{1, \infty}\left(\mathbb{R}^{d}, \mathbb{R}^{d}\right)}<c(\Omega), u=0 \text { on } \partial D\right\},
$$

with $c(\Omega)$ being as in Lemmas 2 and 3. Observing that

$$
\|u\|_{\operatorname{Lip}} \leq\|u\|_{W^{1, \infty}\left(\mathbb{R}^{d}, \mathbb{R}^{d}\right)}
$$

we see that (5) and (6) are satisfied for all $u \in \mathcal{W}$.

It will also be assumed in the sequel that

$$
|\gamma|<\alpha(\epsilon, \mathcal{O}, D) \nu,
$$

where $\alpha$ is furnished by Lemma 1 . The precise value of $\epsilon$ will be fixed below. Now, we choose $g$ satisfying (3) and

$$
g \equiv 0 \text { in a neighborhood of } \mathcal{O} .
$$

(Such a choice is always possible; for instance, one can take $g=a \wedge \nabla \psi$, where $a \in \mathbb{R}^{3}$, $a \cdot \gamma=0,|a|=1, \psi \in C^{\infty}\left(\mathbb{R}^{3}\right), \psi=0$ in $\mathcal{O}, \psi(x)=(g \wedge a) \cdot x$ in a neighborhood of $\partial D$.) If $u \in \mathcal{W}$, one has $g=0$ in a neighborhood of $\partial B+u$. The Navier-Stokes problem in $\Omega+u$ can be written as follows:

$$
\left\{\begin{array}{l}
y(u)-g \in H_{0}^{1}(\Omega+u)^{d}, \\
p(u) \in L^{2}(\Omega+u), \quad \int_{\Omega} p(u) \circ(I+u)=0 \\
-\nu \Delta y(u)+(y(u) \cdot \nabla) y(u)+\nabla p(u)=0 \\
\nabla \cdot y(u)=0 .
\end{array}\right.
$$

From Lemma 1, we know that (8) possesses exactly one solution $(y(u), p(u))$. Accordingly, the drag associated with $B+u$ can be defined and is given by

$$
T(\Omega+u)=\frac{\nu}{2} \int_{\Omega+u} \sigma(y(u))^{2} .
$$

Remark. In principle, it seems more natural to normalize $p(u)$ by imposing that $\int_{\Omega+u} p(u)=0$. However, it will be seen below that the choice that we have made is more useful when one considers different fields $u \in \mathcal{W}$. (Indeed, it yields $\int_{\Omega} P(u)=0$ for the transported pressure $P(u)=p(u) \circ(I+u)$; see (23).) 
4. A differentiability result for the drag. Our main interest in this section to describe the variations of $T(\Omega+u)$ with respect to $u$. As already mentioned in the introduction, we search for a formula

$$
T(\Omega+u)=T(\Omega)+T^{\prime}(\Omega ; u)+o(u),
$$

which must hold for all $u \in \mathcal{W}$, with $T^{\prime}(\Omega ;$. ) being a linear mapping and

$$
\mathcal{o}(u) /\|u\|_{W^{1, \infty}\left(\mathbb{R}^{d}, \mathbb{R}^{d}\right)} \rightarrow 0 \text { as }\|u\|_{W^{1, \infty}\left(\mathbb{R}^{d}, \mathbb{R}^{d}\right)} \rightarrow 0 .
$$

That such a formula can be obtained stems from the next result, which is the most important in this article.

THEOREM 4. There exists $\alpha>0$ such that if $|\gamma|<\alpha \nu$, then $u \mapsto T(\Omega+u)$ is a $C^{\infty}$ mapping in the set $\mathcal{W}$.

In addition, the first derivative at 0 can be obtained from any of the expressions (11), (15), or (18).

THEOREM 5. Assume $|\gamma|<\alpha \nu$.

(i) For all $u \in W^{1, \infty}\left(\mathbb{R}^{d}, \mathbb{R}^{d}\right)$ such that $\left.u\right|_{\partial D}=0$, one has

$T^{\prime}(\Omega ; u)=\nu \int_{\Omega} \sum_{i j} \sigma_{i j}(y)\left(\sigma_{i j}(\dot{y}(u))-\sum_{k}\left(\partial_{i} u_{k} \partial_{k} y_{j}+\partial_{j} u_{k} \partial_{k} y_{i}\right)+\frac{1}{2} \sigma_{i j}(y) \nabla \cdot u\right)$ with $(\dot{y}(u), \dot{p}(u))$ being the unique solution to the linear problem

$$
\left\{\begin{array}{l}
\dot{y}(u) \in H_{0}^{1}(\Omega)^{d}, \\
\dot{p}(u) \in L^{2}(\Omega), \quad \int_{\Omega} \dot{p}(u)=0, \\
-\nu \Delta \dot{y}(u)+(\dot{y}(u) \cdot \nabla) y+(y \cdot \nabla) \dot{y}(u)+\nabla \dot{p}(u)=G(u, y, p), \\
\nabla \cdot \dot{y}(u)=\sum_{i j} \partial_{i} u_{j} \partial_{j} y_{i} .
\end{array}\right.
$$

Here, $y=y(0), p=p(0)$, and $G_{k}(u, y, p) \in H^{-1}(\Omega)$ is given as follows for $1 \leq k \leq d$ :

$$
\begin{aligned}
G_{k}(u, y, p)= & -\nu \sum_{i j}\left(\partial_{j}\left(\partial_{i} u_{j} \partial_{i} y_{k}\right)+\partial_{j}\left(\partial_{j} u_{i} \partial_{i} y_{k}\right)\right)+\nu \sum_{j} \partial_{j}\left((\nabla \cdot u) \partial_{j} y_{k}\right) \\
& +\sum_{i j} y_{i} \partial_{i} u_{j} \partial_{j} y_{k}-(y \cdot \nabla) y_{k} \nabla \cdot u \\
& +\sum_{j} \partial_{j}\left(\partial_{k} u_{j} p\right)-\partial_{k}((\nabla \cdot u) p) .
\end{aligned}
$$

Moreover, $y \in C^{\infty}(\Omega)^{d}, p \in C^{\infty}(\Omega)$, and, consequently,

$$
G(u, y, p)=-\nu \Delta((u \cdot \nabla) y)+(((u \cdot \nabla) y) \cdot \nabla) y+(y \cdot \nabla)((u \cdot \nabla) y)+\nabla(u \cdot \nabla p) .
$$

(ii) One also has

$$
T^{\prime}(\Omega ; u)=\nu \int_{\Omega} \sum_{i j}\left(\sigma_{i j}(y) \sigma_{i j}\left(y^{\prime}(u)\right)+\frac{1}{2} \nabla \cdot\left(\sigma_{i j}(y)^{2} u\right)\right),
$$

with $\left(y^{\prime}(u), p^{\prime}(u)\right)$ being the unique solution to

$$
\left\{\begin{array}{l}
y^{\prime}(u)+(u \cdot \nabla) y \in H_{0}^{1}(\Omega)^{d}, \\
\left(p^{\prime}(u)+u \cdot \nabla p\right) \in L^{2}(\Omega), \quad \int_{\Omega}\left(p^{\prime}(u)+u \cdot \nabla p\right)=0 \\
-\nu \Delta y^{\prime}(u)+\left(y^{\prime}(u) \cdot \nabla\right) y+(y \cdot \nabla) y^{\prime}(u)+\nabla p^{\prime}(u)=0 \\
\nabla \cdot y^{\prime}(u)=0 .
\end{array}\right.
$$


Furthermore, $y^{\prime}(u) \in H_{\mathrm{loc}}^{1}(\Omega)^{d}$ and the sum in (15) satisfies

$$
\sum_{i j}\left(\sigma_{i j}(y) \sigma_{i j}\left(y^{\prime}(u)\right)+\frac{1}{2} \nabla \cdot\left(\sigma_{i j}(y)^{2} u\right)\right) \in L^{1}(\Omega) .
$$

(iii) If $B$ and $D$ are $W^{2, \infty}$ domains and $u \in W^{2, \infty}\left(\mathbb{R}^{d}, \mathbb{R}^{d}\right)$, then $(y, p) \in H^{2}(\Omega)^{d} \times$ $H^{1}(\Omega)$ and

$$
T^{\prime}(\Omega ; u)=\int_{\partial B} u \cdot n\left(\frac{\partial w}{\partial n}-\frac{\partial y}{\partial n}\right) \cdot \frac{\partial y}{\partial n} d s,
$$

with $(w, q)$ being the unique solution to the "adjoint" problem

$$
\left\{\begin{array}{l}
w \in H_{0}^{1}(\Omega)^{d} \cap H^{2}(\Omega)^{d}, \\
q \in H^{1}(\Omega), \quad \int_{\Omega} q=0, \\
-\nu \Delta w_{i}+\sum_{j} \partial_{i} y_{j} w_{j}-\sum_{j} y_{j} \partial_{j} w_{i}+\partial_{i} q=-2 \nu \Delta y_{i}, \quad 1 \leq i \leq d, \\
\nabla . w=0 .
\end{array}\right.
$$

Remark. In order to compute the derivative of the drag in several directions using (15), one has to solve, for each direction $u$, the corresponding partial differential problem (16). It is much more interesting to use the identity (18) because it suffices to solve (2) and (19) only once; then, for each $u$, one has only to compute an integral on $\partial B$.

Remark. One can also obtain expressions for the derivatives of higher orders. This must be made with caution; indeed, $T^{\prime \prime}(\Omega ; .$, . $)$ (i.e., the second derivative at 0 of $u \mapsto T(\Omega+u))$ does not coincide with $\left(T^{\prime}(\Omega ;)^{\prime} ;\right.$. ) (i.e., the derivative at 0 of the mapping $u \mapsto T^{\prime}(\Omega+u$; . )). In fact, these two quantities are related by the following formula (see $[16])$ :

$$
T^{\prime \prime}(\Omega ; u, v)=\left(T^{\prime}(\Omega ; u)^{\prime} ; v\right)-T^{\prime}(\Omega ;(u \cdot \nabla) v) .
$$

5. Differentiability results for the velocity and the pressure. In order to prove Theorem 4, we will first show that $u \mapsto y(u)$ is, in a certain sense, a "differentiable" mapping. An important difficulty arises here, because $y(u)$ is a function defined only for $x \in \Omega+u$, a domain which depends on $u$. This is why we introduce a suitable change of variables and we rewrite the equations satisfied by $y(u)$ and $p(u)$ in the fixed domain $\Omega$. Then, we will have to differentiate the transported variable $Y(u)=y(u) \circ(I+u)$, which is defined in $\Omega$.

In what follows, $y$ and $p$ stand for $y(0)$ and $p(0)$, respectively. We will check the following:

$$
\dot{y}(u)=Y^{\prime}(0) \cdot u \equiv \lim _{t \rightarrow 0} \frac{y(t u) \circ(I+t u)-y}{t} .
$$

This is the "total derivative" of $y(u)$ at 0 , used in (11) to give an expression of $T^{\prime}(\Omega ; u)$. We will also have to use the "local derivative." In fact, we will check that

$$
y^{\prime}(u)=\left.\frac{d}{d v} y(v)\right|_{\omega}(0) \cdot u \equiv \lim _{t \rightarrow 0} \frac{\left.y(t u)\right|_{\omega}-\left.y\right|_{\omega}}{t} \quad \text { in } \omega .
$$


This defines $y^{\prime}(u)$ in each open set $\omega \subset \subset \Omega$ and, consequently, in the whole domain $\Omega$. The previous local derivative was used in (15) to give an expression of $T^{\prime}(\Omega ; u)$. More precisely, the following result holds.

THEOREM 6. There exists $\alpha>0$ such that if $|\gamma|<\alpha \nu$, then

(i) The mapping $u \mapsto(y(u), p(u)) \circ(I+u)$ is $C^{\infty}$ in $\mathcal{W}$, with values in the product space $H^{1}(\Omega)^{d} \times L^{2}(\Omega)$. Its derivative at 0 in the direction $u$ is the unique solution $(\dot{y}(u), \dot{p}(u))$ to $(12)$.

(ii) For all $\omega \subset \subset \Omega$, the mapping $\left.u \mapsto y(u)\right|_{\omega}$ is differentiable in $\mathcal{W}$, with values in $L^{2}(\omega)^{d}$. Its derivative at 0 in the direction $u$ is $\left.y^{\prime}(u)\right|_{\omega}$, where $y^{\prime}(u)$ is uniquely defined by (16). One also has

$$
y^{\prime}(u)=\dot{y}(u)-(u \cdot \nabla) y .
$$

Remark. From general results on local differentiability (see Lemma 2.1 in [15]), (ii) is implied by (i).

Theorems 4, 5, and 6 will be demonstrated in several steps:

- differentiability at 0 of the velocity, the pressure (section 5), and the drag (section 6);

- differentiability at any point in $\mathcal{W}$ (section 7 ); higher-order differentiability (section 8).

6. Proof of differentiability at 0 of the velocity and the pressure. The goal of this section is to prove the following result.

LEMma 7. There exists $\alpha>0$ such that, if $|\gamma|<\alpha \nu$, then the mapping $u \mapsto$ $(y(u), p(u)) \circ(I+u)$, which is defined in $\mathcal{W}$ and takes values in $H^{1}(\Omega)^{d} \times L^{2}(\Omega)$, is differentiable at 0 . Its derivative, denoted by $(\dot{y}(u), \dot{p}(u))$, is uniquely determined by (12).

The proof is based on the implicit function theorem. We will show that this lemma holds with $\alpha$ being of the form $\alpha(\epsilon, \mathcal{O}, D)$ (as in Lemma 1) for an appropriate constant $\epsilon$. First, we will have to rewrite the equations (8) in the fixed domain $\Omega$. For this, we have to "transport" all the terms, some of which belong to $H^{-1}(\Omega+u)$. But it is not clear for a distribution $f \in H^{-1}(\Omega+u)$ how $f \circ(I+u)$ can be defined. Contrarily, following [10, Definition 4.1], one can give a definition of $(f \circ(I+u)) \operatorname{Jac}(I+u)$.

Definition 8. Assume $u \in \mathcal{W}$ and $f \in H^{-1}(\Omega+u)$. Then

$$
(f \circ(I+u)) \operatorname{Jac}(I+u) \in H^{-1}(\Omega)
$$

is defined as follows: for any $\varphi \in H_{0}^{1}(\Omega)$, one has

$$
\langle(f \circ(I+u)) \operatorname{Jac}(I+u), \varphi\rangle_{H^{-1}(\Omega) \times H_{0}^{1}(\Omega)}=\left\langle f, \varphi \circ(I+u)^{-1}\right\rangle_{H^{-1}(\Omega+u) \times H_{0}^{1}(\Omega+u)} .
$$

Remark. Rigorously speaking, $(f \circ(I+u)) \operatorname{Jac}(I+u)$ is not a good notation, because $f \circ(I+u)$ is not defined. However, it will be used in subsequent discussion for convenience.

Note that (21) makes sense; indeed, $\varphi \circ(I+u)^{-1} \in H_{0}^{1}(\Omega+u)$ (see [10, Lemma 4.1]). It does not change the usual definition of $(f \circ(I+u)) \operatorname{Jac}(I+u)$ when $f \in L_{\text {loc }}^{1}(\Omega+u)$.

In order to rewrite (8), we denote by $D(u)$ the operator whose components $D_{i}(u)$ are given as follows:

$$
D_{i}(u)=\sum_{j} M_{i j}(u) \partial_{j}, \quad M(u)={ }^{t}\left[\partial_{j}(I+u)_{i}\right]^{-1} .
$$

Here, ${ }^{t}\left[\partial_{j}(I+u)_{i}\right]^{-1}$ is the transpose of the inverse of the matrix of components $\partial_{j}(I+u)_{i}$. We will use the following three lemmas (see [9] and [10]). 
Lemma 9. Assume $u \in \mathcal{W}$ and $f \in H^{1}(\Omega+u)$. Then

$$
\left(\partial_{i} f\right) \circ(I+u)=\sum_{j} M_{i j}(u) \partial_{j}(f \circ(I+u))=D_{i}(u)(f \circ(I+u)) .
$$

LEMma 10. If $u \in \mathcal{W}$ and $f \in L^{2}(\Omega+u)$, then

$$
\left(\left(\partial_{i} f\right) \circ(I+u)\right) \operatorname{Jac}(I+u)=\sum_{j} \partial_{j}\left(M_{i j}(u)(f \circ(I+u)) \operatorname{Jac}(I+u)\right) .
$$

Lemma 11. Assume $u \in \mathcal{W}$ and $f \in H^{1}(\Omega+u)$. Then

$$
((\Delta f) \circ(I+u)) \operatorname{Jac}(I+u)=\sum_{i j} \partial_{j}\left(M_{i j}(u) \operatorname{Jac}(I+u) D_{i}(u)(f \circ(I+u))\right) .
$$

The Navier-Stokes problem (8) can now be written as follows:

$$
\left\{\begin{array}{l}
Y(u)-g \in H_{0}^{1}(\Omega)^{d}, \\
P(u) \in L^{2}(\Omega), \quad \int_{\Omega} P(u)=0, \\
-\nu \sum_{i j} \partial_{j}\left(M_{i j}(u) \operatorname{Jac}(I+u) D_{i}(u) Y_{k}(u)\right) \\
\quad+(Y(u) \cdot D(u)) Y_{k}(u) \operatorname{Jac}(I+u) \\
\quad+\sum_{j} \partial_{j}\left(M_{k j}(u) P(u) \operatorname{Jac}(I+u)\right)=0, \quad 1 \leq k \leq d, \\
\quad D(u) \cdot Y(u) \operatorname{Jac}(I+u)=0 .
\end{array}\right.
$$

Here, we have set $Y(u)=y(u) \circ(I+u)$ and $P(u)=p(u) \circ(I+u)$.

We will also introduce in (23) the new variable $X(u)=Y(u)-g$. This leads to the following system, equivalent to (23) (which is, in turn, equivalent to (8)):

$$
\left\{\begin{array}{l}
X(u) \in H_{0}^{1}(\Omega)^{d}, \\
P(u) \in L^{2}(\Omega), \quad \int_{\Omega} P(u)=0, \\
-\nu \sum_{i j} \partial_{j}\left(M_{i j}(u) \operatorname{Jac}(I+u) D_{i}(u)(X(u)+g)_{k}\right) \\
\quad+((X(u)+g) \cdot D(u))(X(u)+g)_{k} \operatorname{Jac}(I+u) \\
\quad+\sum_{j} \partial_{j}\left(M_{k j}(u) P(u) \operatorname{Jac}(I+u)\right)=0, \quad 1 \leq k \leq d, \\
\quad D(u) \cdot(X(u)+g) \operatorname{Jac}(I+u)=0 .
\end{array}\right.
$$

This equation can be written

$$
H(u ; X(u), P(u))=0,
$$

where the function $H$ is defined, from $\mathcal{W} \times H_{0}^{1}(\Omega)^{d} \times L_{0}^{2}(\Omega)$ into $H^{-1}(\Omega)^{d} \times L_{0}^{2}(\Omega)$, by

$$
\left\{\begin{aligned}
H(u ; \chi, \pi)=(F(u ; \chi, \pi), R(u ; \chi, \pi)), \quad F=\left(F_{1}, \ldots, F_{d}\right) \\
\begin{array}{rl}
F_{k}(u ; \chi, \pi)=-\nu & \sum_{i j} \partial_{j}\left(M_{i j}(u) \operatorname{Jac}(I+u) D_{i}(u)(\chi+g)_{k}\right) \\
& \quad+((\chi+g) \cdot D(u))(\chi+g)_{k} \operatorname{Jac}(I+u) \\
& \quad+\sum_{j} \partial_{j}\left(M_{k j}(u) \pi \operatorname{Jac}(I+u)\right), \quad 1 \leq k \leq d
\end{array} \\
R(u ; \chi, \pi)=D(u) \cdot(\chi+g) \operatorname{Jac}(I+u) .
\end{aligned}\right.
$$


The fact that $R(u ; \chi, \pi) \in L_{0}^{2}(\Omega)$ is crucial. This is true because

$$
\begin{aligned}
\int_{\Omega}(D(u) \cdot Y(u)) \operatorname{Jac}(I+u) & =\int_{\Omega+u}(D(u) \cdot Y(u)) \circ(I+u)^{-1} \\
& =\int_{\Omega+u} \nabla \cdot\left(Y(u) \circ(I+u)^{-1}\right) \\
& =0 .
\end{aligned}
$$

Now, we check that the assumptions of the implicit function theorem are satisfied. First, $H$ is $C^{1}$ in a neighborhood of $(0 ; X, P)$, where we have set $X=X(0)=y-g$, $P=P(0)=p$. Indeed, the coefficients in $D(u)$ and $M(u)$ are $C^{1}$ since, according to the results in [10], the mapping $u \mapsto M_{i j}(u)$ is $C^{1}$ in a neighborhood of 0 in $W^{1, \infty}\left(\mathbb{R}^{d}, \mathbb{R}^{d}\right)$, with values in $L^{\infty}\left(\mathbb{R}^{d}, \mathbb{R}^{d^{2}}\right)$.

On the other hand, let us see that the differential operator $L=D_{(\chi, \pi)} H(0 ; X, P)$ is an isomorphism from $H_{0}^{1}(\Omega)^{d} \times L_{0}^{2}(\Omega)$ onto $H^{-1}(\Omega)^{d} \times L_{0}^{2}(\Omega)$. For each $(\chi, \pi) \in$ $H_{0}^{1}(\Omega)^{d} \times L_{0}^{2}(\Omega)$, one has

$$
L(\chi, \pi)=(-\nu \Delta \chi+(\chi \cdot \nabla) y+(y \cdot \nabla) \chi+\nabla \pi, \nabla \cdot \chi) .
$$

The operator $L$ is linear and bounded from $H_{0}^{1}(\Omega)^{d} \times L_{0}^{2}(\Omega)$ into $H^{-1}(\Omega)^{d} \times L_{0}^{2}(\Omega)$. Hence, we have to check that, for each $f \in H^{-1}(\Omega)^{d}$ and $\phi \in L_{0}^{2}(\Omega)$, there exists a unique solution $(\chi, \pi) \in H_{0}^{1}(\Omega)^{d} \times L_{0}^{2}(\Omega)$ to the system

$$
\left\{\begin{array}{l}
-\nu \Delta \chi+(\chi \cdot \nabla) y+(y \cdot \nabla) \chi+\nabla \pi=f, \\
\nabla \cdot \chi=\phi
\end{array}\right.
$$

and, also, that this solution depends continuously on the data. Since $\Omega$ is a Lipschitz domain, Corollary 2.4 in [6] asserts

$$
\forall \phi \in L^{2}(\Omega) \text { such that } \int_{\Omega} \phi=0, \text { there exists } \psi \in H_{0}^{1}(\Omega)^{d} \text { such that } \nabla \cdot \psi=\phi \text {. }
$$

Setting $\Phi=\chi-\psi$, system (28) reduces to

$$
\left\{\begin{array}{l}
\Phi \in V, \quad \pi \in L_{0}^{2}(\Omega), \\
-\nu \Delta \Phi+(\Phi \cdot \nabla) y+(y \cdot \nabla) \Phi+\nabla \pi=F,
\end{array}\right.
$$

where $V=\left\{v \in H_{0}^{1}(\Omega)^{d} ; \nabla \cdot v=0\right\}$ and $F=f+\nu \Delta \psi-(\psi \cdot \nabla) y-(y \cdot \nabla) \psi$. This equation is elliptic with respect to $\Phi$ and possesses a unique solution depending continuously on the data if, for some appropriate $r=r(\mathcal{O}, D)>0$, one has

$$
\|y\|_{H_{0}^{1}(\Omega)^{d}}<r \nu .
$$

Hence, if we choose $\epsilon<r, \alpha=\alpha(\epsilon, \mathcal{O}, D)$ as in Lemma 1 and

$$
|\gamma|<\alpha \nu,
$$

this condition holds and $L$ is an isomorphism.

This allows us to apply the implicit function theorem to $(25)$. We deduce that the mapping $u \mapsto(X(u), P(u))$, which takes values in the space $H_{0}^{1}(\Omega)^{d} \times L_{0}^{2}(\Omega)$, is 
differentiable at 0 . Since $y(u) \circ(I+u)=X(u)+g$ and $p(u) \circ(I+u)=P(u)$, the first part of Lemma 7 is proven.

Finally, let us deduce the equations satisfied by $(\dot{y}(u), \dot{p}(u))$. In accordance with the implicit function theorem,

$$
L(\dot{y}(u), \dot{p}(u))=-D_{v} H(0 ; X, P) \cdot u
$$

for all admissible $u$. Taking into account (26) and also the identities

$$
M_{i k}^{\prime}(0) \cdot u=-\partial_{i} u_{k} \quad \text { and } \quad \frac{d}{d v} \operatorname{Jac}(I+v)(0) \cdot u=\nabla \cdot u
$$

(see [10]), we find that $(\dot{y}(u), \dot{p}(u))$ is a solution to (12). But this problem possesses exactly one solution, since $L$ is an isomorphism. Consequently, Lemma 7 is proven.

Remark. In order to solve (28), we have had to assume that $\Omega$ is a Lipschitz domain. The same requirement is found when one writes (28) as a mixed problem and one tries to apply general results concerning mixed variational formulations.

7. Proof of differentiability at $\mathbf{0}$ of the drag. The goal of this section is to prove Theorem 5 .

Proof of part (i). By definition, one has

$$
\begin{aligned}
T(\Omega+u) & =\frac{\nu}{2} \int_{\Omega+u} \sum_{i j}\left(\partial_{i} y_{j}(u)+\partial_{j} y_{i}(u)\right)^{2} \\
& =\frac{\nu}{2} \int_{\Omega} \sum_{i j}\left(\sum_{k}\left(M_{i k}(u) \partial_{k} Y_{j}(u)+M_{j k}(u) \partial_{k} Y_{i}(u)\right)\right)^{2} \operatorname{Jac}(I+u) .
\end{aligned}
$$

We will deduce the differentiability of the mapping $u \mapsto T(\Omega+u)$ from the following result (Theorem 4.1 in [10]).

Lemma 12. Assume that $z(u)$ is well defined for all $u \in \mathcal{W}$ and, also, that

$$
u \mapsto z(u) \circ(I+u) \text { is differentiable at } 0 \text {, with values in } L^{1}(\Omega) \text {. }
$$

Then the mapping $u \mapsto S(\Omega+u)=\int_{\Omega}(z(u) \circ(I+u)) \operatorname{Jac}(I+u)$ is also differentiable at 0 . Its derivative at 0 in the direction $u$ is given by

$$
S^{\prime}(\Omega ; u)=\int_{\Omega}(\dot{z}(u)+z(0) \nabla \cdot u) .
$$

We will apply this lemma with

$$
z(u) \circ(I+u)=\sum_{i j}\left(\sum_{k}\left(M_{i k}(u) \partial_{k} Y_{j}(u)+M_{j k}(u) \partial_{k} Y_{i}(u)\right)\right)^{2} .
$$

Obviously, $S(\Omega+u) \equiv T(\Omega+u)$ in this case; also, that (32) holds is deduced from the differentiability at 0 of the $H_{0}^{1}(\Omega)^{d}$-valued mapping $u \mapsto Y(u)$.

Let us compute $T^{\prime}(\Omega ; u)$. From (31) and the fact that $M(0)=I d$, one has

$$
\begin{aligned}
\dot{z}(u) & =2 \sum_{i j}\left(\partial_{i} y_{j}+\partial_{j} y_{i}\right)\left(\partial_{i} \dot{y}_{j}(u)+\partial_{j} \dot{y}_{i}(u)-\sum_{k} \partial_{i} u_{k} \partial_{k} y_{j}-\sum_{k} \partial_{j} u_{k} \partial_{k} y_{i}\right) \\
& =2 \sum_{i j} \sigma_{i j}(y)\left(\sigma_{i j}(\dot{y}(u))-\sum_{k} \partial_{i} u_{k} \partial_{k} y_{j}-\sum_{k} \partial_{j} u_{k} \partial_{k} y_{i}\right) .
\end{aligned}
$$

Since $z(0)=\sum_{i j} \sigma_{i j}(y)^{2}$, we have

$T^{\prime}(\Omega ; u)=\nu \int_{\Omega} \sum_{i j} \sigma_{i j}(y)\left(\sigma_{i j}(\dot{y}(u))-\sum_{k}\left(\partial_{i} u_{k} \partial_{k} y_{j}+\partial_{j} u_{k} \partial_{k} y_{i}\right)+\frac{1}{2} \sigma_{i j}(y) \nabla \cdot u\right)$. 
This proves (11). The regularity results are $y \in C^{\infty}(\Omega)^{d}$ and $p \in C^{\infty}(\Omega)$. (This is well known; for instance, see [7].) The identity (14) is then an easy consequence of (13).

Proof of part (ii). Let us set

$$
y^{\prime}(u)=\dot{y}(u)-(u \cdot \nabla) y, \quad p^{\prime}(u)=\dot{p}(u)-u \cdot \nabla p .
$$

Using (14) we see that (12) and (16) are equivalent. On the other hand, these definitions provide the following identity:

$$
\begin{aligned}
\sum_{i j} & \left(\sigma_{i j}(y) \sigma_{i j}\left(y^{\prime}(u)\right)+\frac{1}{2} \nabla \cdot\left(\sigma_{i j}(y)^{2} u\right)\right) \\
& =\sum_{i j} \sigma_{i j}(y)\left(\sigma_{i j}(\dot{y}(u))-\sum_{k}\left(\partial_{i} u_{k} \partial_{k} y_{j}+\partial_{j} u_{k} \partial_{k} y_{i}\right)+\frac{1}{2} \sigma_{i j}(y) \nabla \cdot u\right) .
\end{aligned}
$$

Hence, (11) implies (17) and (15).

Proof of part (iii). Let us now suppose that $\Omega$ is a $W^{2, \infty}$ domain and $u \in$ $W^{2, \infty}\left(\mathbb{R}^{d}, \mathbb{R}^{d}\right)$. According to Lemma 1 , one has $y \in H^{2}(\Omega)^{d}$ and $p \in H^{1}(\Omega)$. Consequently, one obtains from (15)

$$
T^{\prime}(\Omega ; u)=\nu \int_{\Omega} \sum_{i j} \sigma_{i j}(y) \sigma_{i j}\left(y^{\prime}(u)\right)+\frac{\nu}{2} \int_{\partial \Omega} \sum_{i j} \sigma_{i j}(y)^{2} u \cdot n d s .
$$

Since $\dot{y}(u)=0$ and $y \equiv$ const. on $\partial \Omega, y^{\prime}(u)=-u \cdot n \frac{\partial y}{\partial n}$ on $\partial \Omega$. Therefore,

$$
\begin{aligned}
\nu \int_{\Omega} \sum_{i j} \sigma_{i j}(y) & \sigma_{i j}\left(y^{\prime}(u)\right) \\
& =-2 \nu \int_{\Omega} \Delta y \cdot y^{\prime}(u)-2 \nu \sum_{i j} \int_{\partial \Omega} u \cdot n\left(\partial_{i} y_{j}+\partial_{j} y_{i}\right) \frac{\partial y_{i}}{\partial n} n_{j} d s .
\end{aligned}
$$

In addition, $\sum_{i} \partial_{i} y_{i}=0$ imply $\sum_{i j}\left(\partial_{i} y_{j}+\partial_{j} y_{i}\right) \frac{\partial y_{i}}{\partial n} n_{j}=\left|\frac{\partial y}{\partial n}\right|^{2}$, whence

$$
T^{\prime}(\Omega ; u)=-2 \nu \int_{\Omega} \Delta y \cdot y^{\prime}(u)-\nu \int_{\partial \Omega}\left|\frac{\partial y}{\partial n}\right|^{2} u \cdot n d s .
$$

If $w$ and $q$ are given by (19), after some manipulation, one obtains

$$
\begin{aligned}
T^{\prime}(\Omega ; u)=\int_{\Omega} \sum_{i}\left(-\nu \Delta w_{i} y_{i}^{\prime}(u)+\sum_{j}\left(\partial_{i} y_{j} w_{j}\right.\right. & \left.\left.-y_{j} \partial_{j} w_{i}\right) y_{i}^{\prime}(u)+\partial_{i} q y_{i}^{\prime}(u)\right) \\
& -\nu \int_{\partial \Omega}\left|\frac{\partial y}{\partial n}\right|^{2} u \cdot n d s \\
=\left\langle-\nu \Delta y^{\prime}(u)+\left(y^{\prime}(u) \cdot \nabla\right) y+(y \cdot \nabla)\right. & \left.y^{\prime}(u)+\nabla p^{\prime}(u), w\right\rangle_{H^{-1}(\Omega)^{d} \times H_{0}^{1}(\Omega)^{d}} \\
& +\nu \int_{\partial \Omega} u \cdot n\left(\frac{\partial w}{\partial n}-\frac{\partial y}{\partial n}\right) \cdot \frac{\partial y}{\partial n} d s .
\end{aligned}
$$

Using (16) satisfied by $\left(y^{\prime}(u), p^{\prime}(u)\right)$, one sees that the duality product on the righthand side cancels. This proves (18), since $u=0$ on $\partial D$. 
8. Proof of differentiability at any point in $\mathcal{W}$ of the velocity, the pressure, and the drag. In this section, we prove the following result.

LEMMA 13. The mapping $u \mapsto(y(u), p(u)) \circ(I+u)$, which takes values in $H^{1}(\Omega)^{d} \times L^{2}(\Omega)$, is differentiable at any point $u_{0} \in \mathcal{W}$. The mapping $u \mapsto T(\Omega+u)$ is also differentiable at any $u_{0} \in \mathcal{W}$.

Proof. Let $u_{0} \in \mathcal{W}$ be given. We have

$$
\Omega+\left(u_{0}+v\right)=\left(\Omega+u_{0}\right)+v \circ\left(I+u_{0}\right)^{-1}
$$

for $v \in \mathcal{W}$ small enough in order to have $u_{0}+v \in \mathcal{W}$. According to the results in section 6 , the mapping $w \mapsto T\left(\left(\Omega+u_{0}\right)+w\right)$ is differentiable at 0 . The mapping $v \mapsto v \circ\left(I+u_{0}\right)^{-1}$ is linear and bounded (therefore differentiable) from $W^{1, \infty}\left(\mathbb{R}^{d}, \mathbb{R}^{d}\right)$ into itself. Consequently,

$$
v \mapsto T\left(\left(\Omega+u_{0}\right)+v \circ\left(I+u_{0}\right)^{-1}\right) \quad \text { is differentiable at } 0 ;
$$

i.e., $u \mapsto T(\Omega+u)$ is differentiable at $u_{0}$.

Now we will apply the previous results to some new reference domains different from $\Omega$. So we introduce the more explicit notation $(y(\Omega ; v), p(\Omega ; v))$ for the solution to the Navier-Stokes problem in $\Omega+v$. We see from (34) that, for small $v$,

$$
y\left(\Omega ; u_{0}+v\right) \circ\left(I+\left(u_{0}+v\right)\right)=y\left(\Omega+u_{0} ; v \circ\left(I+u_{0}\right)^{-1}\right) \circ\left(I+u_{0}+v\right) .
$$

On the other hand, from Lemma 7, we know that the $H^{1}\left(\Omega+u_{0}\right)^{d}$-valued mapping $w \mapsto y\left(\Omega+u_{0} ; w\right) \circ(I+w)$ is differentiable at 0 . Thus, $v \mapsto y\left(\Omega ; u_{0}+v\right) \circ\left(I+u_{0}+v\right)$ is differentiable at 0 ; i.e., $u \mapsto y(\Omega ; u) \circ(I+u)$ is differentiable at $u_{0}$. A similar argument holds for the function $u \mapsto p(\Omega ; u) \circ(I+u)$.

Remark. Theorem 4.1 in [1] asserts that, when $\Omega$ is a $W^{2, \infty}$ domain, the mapping $u \mapsto(y(u), p(u)) \circ(I+u)$ is well defined for $u \in W^{2, \infty}\left(\mathbb{R}^{d}, \mathbb{R}^{d}\right) \cap \mathcal{W}$ and differentiable at 0 , with values in $H^{2}(\Omega)^{d} \times H^{1}(\Omega)$. Adapting the previous argument, we can deduce differentiability at each point in a $W^{2, \infty}$-open ball centered at 0 .

9. Higher-order differentiability. In this section, we will prove Theorems 6 and 4 .

Proof of part (i) of Theorem 6. It remains to prove that $u \mapsto(Y(u), P(u))$ is a $C^{\infty}$ mapping. (The remainder of part (i) has already been proven in section 6 , Lemma 7 .)

Observe that the mapping $H$, introduced in section 5 and defined from $\mathcal{W} \times$ $H_{0}^{1}(\Omega)^{d} \times L_{0}^{2}(\Omega)$ into $H^{-1}(\Omega)^{d} \times L_{0}^{2}(\Omega)$, is $C^{\infty}$. This is a consequence of the fact that $u \mapsto M_{i j}(u)$ and $u \mapsto \operatorname{Jac}(I+u)$ are $C^{\infty}$ mappings. In turn, this stems from the following:

(a) The mapping $u \mapsto \operatorname{Jac}(I+u)$ is multilinear and, consequently, is of class $C^{\infty}$.

(b) The mapping $u \mapsto M(u)={ }^{t}\left[\partial_{i}(I+u)_{j}\right]^{-1}$ is $C^{\infty}$ on $\mathcal{W}$, because the inversion operator is indefinitely differentiable in the set of the nonsingular matrices.

From the implicit function theorem, we deduce that $u \mapsto(Y(u), P(u))$ possesses derivatives of all orders at 0. Again using (35), which can be written in the form

$$
Y\left(\Omega ; u_{0}+u\right)=Y\left(\Omega+u_{0} ; u \circ\left(I+u_{0}\right)^{-1}\right) \circ\left(I+u_{0}\right),
$$

one also sees that $u \mapsto Y(\Omega ; u)$ is $C^{\infty}$ at each point $u_{0} \in \mathcal{W}$. The same is true for $u \mapsto P(\Omega ; u)$.

Proof of part (ii). The differentiability of the mapping $\left.u \mapsto y(u)\right|_{\omega}$ at 0 in $L^{2}(\omega)^{d}$ and the identity (20) are consequences of the differentiability of $u \mapsto y(u) \circ(I+u)$ 
given in Lemma 7. This is a consequence of general results on differentiation with respect to domains (see Lemma 2.1 in [15]). On the other hand, (12) and (20) together imply (16).

Proof of Theorem 4 . We have to check that $u \mapsto T(\Omega+u)$ is a $C^{\infty}$ mapping. This is deduced from the above results and the following equality, which has already been used in section 6 :

$T(\Omega+u)=\frac{\nu}{2} \int_{\Omega} \sum_{i j}\left(\sum_{k}\left(M_{i k}(u) \partial_{k} Y_{j}(\Omega ; u)+M_{j k}(u) \partial_{k} Y_{i}(\Omega ; u)\right)\right)^{2} \operatorname{Jac}(I+u)$.

10. Miscellaneous remarks. The case of a non-Lipschitz domain. Until now, we have assumed that $\Omega$ is a Lipschitz domain in order to ensure, among other things, that (29) is true. Actually, this assumption on $\Omega$ can be replaced by (29) itself:

$\forall \phi \in L^{2}(\Omega)$ such that $\int_{\Omega} \phi=0$, there exists $\psi \in H_{0}^{1}(\Omega)^{d}$ such that $\nabla \cdot \psi=\phi ;$

i.e., the divergence operator maps $H_{0}^{1}(\Omega)^{d}$ onto $L_{0}^{2}(\Omega)$.

Under this weaker hypothesis, the results in the previous sections hold again with minor changes. Instead of $p \in C^{\infty}(\Omega) \cap L^{2}(\Omega)$, we now have only

$$
p \in C^{\infty}(\Omega), \quad \nabla p \in H^{-1}(\Omega)^{d} .
$$

On the other hand, we cannot normalize $p$ and $\dot{p}(u)$ as before. Instead, a possibility is to fix a nonempty open set $\omega \subset \subset \Omega$ and to impose

$$
\int_{\omega} p=0, \quad \int_{\omega} \dot{p}(u)=0 .
$$

Remark. The condition (29) requires some regularity on $\Omega$, which is probably not far from being Lipschitz.

Remark. It is important to note that, here, the difficulty is not related to nonlinearity. Even if we were concerned with Stokes flows (the term $(y \cdot \nabla) y$ disappears), (36) could not be improved unless a regularity assumption is required for $\Omega$. This difficulty is connected with the fact that the equations are coupled by the incompressibility condition $\nabla \cdot y=0$.

Remark. For more simple (scalar) problems, we can obtain a result similar to Theorem 4 , without any regularity hypothesis for $\Omega$. For example, let $y$ be the unique solution to

$$
-\Delta y=f \text { in } \Omega, \quad y-g \in H_{0}^{1}(\Omega)^{d},
$$

and let us set

$$
S(\Omega)=\int_{\Omega}|\nabla(y-z)|^{2},
$$

where $f \in L^{2}(\Omega)^{d}, g \in H^{2}\left(\mathbb{R}^{d}\right)$, and $z \in H^{1}\left(\mathbb{R}^{d}\right)$ are given and $\Omega$ is an arbitrary bounded open set in $\mathbb{R}^{d}$. Then, $u \mapsto S(\Omega+u)$ is well defined and differentiable in a neighborhood of 0 in $W^{1, \infty}\left(\mathbb{R}^{d}, \mathbb{R}^{d}\right)[10$, Theorem 5.2, p. V.10].

The particular case of a polygonal two-dimensional body. Assume that $B$ is a twodimensional polygonal domain with vertices $s_{1}, s_{2}, \ldots, s_{n}$. Let us set $s=\left(s_{1}, \ldots, s_{n}\right)$, 
and let us assume that the corresponding polygonal line, $\partial B$, does not cross itself. Thus, using the notation $s_{n+1}=s_{1}$, one has

$$
\left[s_{i}, s_{i+1}\left[\bigcap \left[s_{j}, s_{j+1}[=\emptyset \text { if } 1 \leq i<j \leq n .\right.\right.\right.
$$

Also, assume that

$$
B \subset \subset \mathcal{O} \subset \subset D
$$

It is then obvious that $\Omega_{s}=D \backslash \bar{B}$ satisfies (1). In this situation, the following is not difficult to prove:

The mapping $s \mapsto T\left(\Omega_{s}\right)$ is $C^{\infty}$ at each point $s \in \mathbb{R}^{2 n}$ satisfying (38) and (39).

Other examples. Above, the polygonal domain can be replaced by a spline depending on a finite number of parameters. In such a way, we obtain similar results for "NACA profiles" or other piecewise $C^{1}$ boundaries. Similar results hold for threedimensional domains.

11. Appendix. In order to prove Lemma 3, we need some previous definitions and results.

DEFINITION 14. Let $\Omega$ be a bounded open set in $\mathbb{R}^{d}$.

(i) We say that $\Omega$ is a Lipschitz domain (also that $\Omega$ is Lipschitzian; see [11], [5]) if there exist constants $a>0$ and $b>0$ such that, for each $z \in \partial \Omega$, one can find

- coordinates $\left(x_{1}, \ldots, x_{d}\right)$,

- a Lipschitz-continuous real-valued function $\psi$ in $\Theta_{*}$ with best Lipschitz constant smaller than $b$, where $\Theta_{*}=\left\{x_{*} ;\left|x_{*}-z_{*}\right|<a\right\}, x_{*}=\left(x_{1}, \ldots, x_{d-1}\right)$, and $z_{*}=$ $\left(z_{1}, \ldots, z_{d-1}\right)$,

such that, for each $x \in \Theta=\left\{x \in \mathbb{R}^{d} ;\left|x_{*}-z_{*}\right|<a,\left|x_{d}-\psi\left(x_{*}\right)\right|<a\right\}$, one has

$$
x \in \Omega \Longleftrightarrow x_{d}>\psi\left(x_{*}\right) .
$$

(ii) We say that $\Omega$ satisfies the cone property uniformly if there exist constants $\alpha>0$ and $b>0$ such that, for each $z \in \partial \Omega$, one can find coordinates such that

$$
x \in \Omega \cap B(z ; \alpha) \Longrightarrow x+\mathcal{C}_{b, \alpha} \subset \Omega .
$$

Here, we have set $B(z ; \alpha)=\left\{x \in \mathbb{R}^{d} ;|x-z|<\alpha\right\}$ and

$$
\mathcal{C}_{b, \alpha}=\left\{x \in \mathbb{R}^{d} ; x_{d}>b\left|x_{*}\right|,|x|<\alpha\right\} .
$$

The properties (i) and (ii) are equivalent. More precisely, we have the following result (see [3]).

LEMma 15. A bounded open set in $\mathbb{R}^{d}$ is Lipschitzian if and only if it satisfies the cone property uniformly.

The following result was also used in the proof of Lemma 3.

Lemma 16. Assume that $\alpha>0$ and $b>0$ are given. There exist $\alpha^{\prime}>0$, $b^{\prime}>0$, and $l \in(0,1)$ such that, whenever $v \in W^{1, \infty}\left(\mathbb{R}^{d}, \mathbb{R}^{d}\right),\|v\|_{W^{1, \infty}\left(\mathbb{R}^{d}, \mathbb{R}^{d}\right)} \leq l$, and $v(0)=0$, one has

$$
\mathcal{C}_{b^{\prime}, \alpha^{\prime}} \subset(I+v) \mathcal{C}_{b, \alpha} .
$$

Proof of Lemma 3. From Lemma 15, there exist $\alpha>0$ and $b>0$ such that, for each $z \in \partial \Omega$, one has

$$
x \in \Omega \cap B(z ; \alpha) \Longrightarrow x+\mathcal{C}_{b, \alpha} \subset \Omega .
$$


Again from Lemma 15, it is enough to find $\alpha^{\prime}$ and $b^{\prime}$ such that, for each $z^{\prime} \in \partial(\Omega+u)$,

$$
x^{\prime} \in(\Omega+u) \cap B\left(z^{\prime} ; \alpha^{\prime}\right) \Longrightarrow x^{\prime}+\mathcal{C}_{b^{\prime}, \alpha^{\prime}} \subset \Omega+u .
$$

Given such an $x^{\prime}$, let $\xi^{\prime} \in \mathcal{C}_{b^{\prime}, \alpha^{\prime}}$, and define $x$ and $z$ by $x^{\prime}=x+u(x), z^{\prime}=z+u(z)$. Lemma 16 with $v(\xi)=u(\xi+x)-u(x)$ gives the existence of $\xi \in \mathcal{C}_{b, \alpha}$ such that $\xi^{\prime}=\xi+u(\xi+x)-u(x)$. Then

$$
x^{\prime}+\xi^{\prime}=x+\xi+u(x+\xi) .
$$

This gives (41), provided that $x+\xi \in \Omega$. By (40), it is enough to check that $x \in \Omega$ (which is obvious) and $|x-z| \leq \alpha$, which is satisfied for $\alpha^{\prime} \leq \alpha(1-c)$ (indeed, $x^{\prime}-z^{\prime}=x-z+u(x)-u(z)$ implies $\left.\left|x^{\prime}-z^{\prime}\right| \geq|x-z|(1-c)\right)$.

\section{REFERENCES}

[1] J.A. Bello, E. Fernández-Cara, and J. Simon, Optimal shape design for Navier-Stokes flow, in System Modelling and Optimization, Lecture Notes in Control and Inform. Sci. 180, P. Kall, ed., Springer-Verlag, Berlin, 1992, pp. 481-489.

[2] J.A. Bello, E. Fernández-Cara, and J. Simon, Variation par rapport au domaine de l'énergie visqueuse dissipée dans un fluide de Navier-Stokes, C. R. Acad. Sci. Paris Sér. I Math., 313 (1991), pp. 447-450.

[3] D. Chenais, Un résultat de compacité d'un ensemble de parties de $\mathbb{R}^{n}, \mathrm{C}$. R. Acad. Sci. Paris Sér. A, 277 (1973), pp. 905-907.

[4] R. Dautray and J.L. Lions, Analyse mathématique et calcul numérique pour les sciences et les techniques, tome 1, Masson, Paris, 1984.

[5] D. Gilbarg and N.S. Trudinger, Elliptic Partial Differential Equations of Second Order, Springer-Verlag, Berlin, 1983.

[6] V. Girault and P.A. Raviart Finite Element Methods for Navier-Stokes Equations, Springer-Verlag, Berlin, 1983.

[7] O.A. Ladyzhenskaya, The Mathematical Theory of Viscous Incompressible Flow, Gordon and Breach, New York, 1969.

[8] J. Lemoine, Couplage et contrôle pour les équations de Navier-Stokes: Espaces fonctionnels, Thesis, Université Blaise Pascal (Clermont-Ferrand 2), 1995.

[9] F. Murat And J. Simon, Quelques résultats sur le contrôle par un domaine géométrique, Report of L.A. 189 74003, Université Paris VI, 1974.

[10] F. Murat and J. Simon, Sur le contrôle par un domaine géométrique, Report of L.A. 189 76015, Université Paris VI, 1976.

[11] J. NEČAS, Les méthodes directes en théorie des équations elliptiques, Masson, Paris, 1967.

[12] O. Pironneau, On optimum design in fluid mechanics, J. Fluid. Mech., 64 (1974), pp. 97-110.

[13] O. Pironneau, Optimal Shape Design for Elliptic Systems, Springer-Verlag, New York, 1984.

[14] H. Schlichting, Boundary Layer Theory, Academic Press, New York, 1970.

[15] J. Simon, Differentiation with respect to the domain in boundary value problems, Numer. Funct. Anal. Optim., 2 (1980), pp. 649-687.

[16] J. Simon, Second variation in domain optimization problems, in Control and Estimation of Distributed Parameter Systems, Internat. Ser. Numer. Math. 91, F. Kappel, K. Kunisch, and W. Schappacher, eds., Birkhäuser, Basel, 1989, pp. 361-378.

[17] J. Simon, Domain variation for drag in Stokes flow, in Control Theory of Distributed Parameter Systems and Applications, Lecture Notes in Control and Inform. Sci. 159, X. Li and J. Yong, eds., Springer-Verlag, Berlin, 1991, pp. 28-42. 